\title{
Capsule Commentary on Franklin et al., Time to Filling of New Prescriptions for Chronic Disease Medications Among a Cohort of Elderly Patients in the USA
}

\author{
John-Michael Gamble, PhD \\ School of Pharmacy, Faculty of Science, University of Waterloo, Waterloo, ON, Canada.
}

$\mathrm{J}$ Gen Intern Med 33(11): 1955

DOI: $10.1007 / \mathrm{s} 11606-018-4642-0$

() Society of General Internal Medicine 2018

$\mathrm{W}$ hen a patient forgoes filling a new prescription, this is known as primary medication non-adherence. Medication non-adherence is costly to the healthcare system, associated with increased hospitalization and worse patient health outcomes. ${ }^{1}$ It is difficult to study, given that direct observation of medication consumption is generally not feasible. However, Franklin et al. ${ }^{2}$ expand our limited understanding of prescription-filling behavior through conducting a cohort study using linked US-based healthcare databases that contain both prescribing data from physician records and dispensation data.

Franklin et al. found that about $20 \%$ of new prescriptions for adults over 65 years of age went unfilled within 30 days (25\% within 7 days and $9 \%$ within 1 year) of receiving a prescription to manage common chronic diseases (diabetes, hypertension, dyslipidemia, asthma/COPD, and osteoporosis) with no significant differences between therapeutic classes. This magnitude of primary non-adherence is marginally lower compared to that of prior studies. Others have also found substantial variation across therapeutic classes. ${ }^{3,4}$ Researchers should continue to study variation across therapeutic areas and variation within a patient's treatment course.

Using several clever analytic approaches, Franklin et al. give us two key variables for identifying patients at risk of not filling their new prescription: number of current medications and out-of-pocket costs. Although they assessed a suite of covariates as predictors for primary non-adherence and had higher predictive accuracy than previous studies, factors affecting prescription-filling behavior such as fear of adverse effects, uncertainty of benefit, and the patient's rationale for choosing not to fill their prescription were not measured. Other important chronic diseases were not considered such as depression, heart failure, rheumatoid arthritis, and chronic kidney disease, limiting the generalizability of their findings. Others have recently found that the mode of prescription (electronic vs. paper) impacts the prevalence of primary nonadherence. $^{5}$

What are the take-home points for health care practitioners? First, one out of five new prescriptions for common chronic conditions may go unfilled. Second, when prescribing a new medication for hypertension, diabetes, osteoporosis, or dyslipidemia to a patient who is not taking any other medications and required a co-pay of over $\$ 5$, additional follow-up is prudent.

Corresponding Author: John-Michael Gamble, $\mathrm{PhD}$; School of Pharmacy, Faculty of Science University of Waterloo, Waterloo, ON, Canada (e-mail:jm.gamble@uwaterloo.ca).

\section{Compliance with Ethical Standards:}

Conflict of Interest: The author declares that he does not have a conflict of interest.

\section{REFERENCES}

1. Simpson SH, Eurich DT, Majumdar SR, et al. A meta-analysis of the association between adherence to drug therapy and mortality. BMJ 2006;333(7557):15-18.

2. Franklin JM, Mahesri M, Krumme AA, et al. Time to filling of new prescriptions for chronic disease medications among a cohort of elderly patients in the USA. J Gen Intern Med. 2018;1-8. https://doi.org/10. 1007/s11606-018-4592-6

3. Tamblyn R, Eguale T, Huang A, Winslade N, Doran P. The incidence and determinants of primary nonadherence with prescribed medication in primary care: a cohort study. Ann Intern Med 2014;160(7):441. https:// doi.org/10.7326/M13-1705

4. Raebel MA, Ellis JL, Carroll NM, et al. Characteristics of Patients with Primary Non-adherence to Medications for Hypertension, Diabetes, and Lipid Disorders. J Gen Intern Med 2012;27(1):57-64. https://doi.org/10. 1007/s11606-011-1829-Z

5. Adamson AS, Suarez EA, Gorman AR. Association between method of prescribing and primary nonadherence to dermatologic medication in an urban hospital population. JAMA Dermatol 2017;153(1):49-54. https:// doi.org/10.1001/jamadermatol.2016.3491 